\title{
CEt KRAS Antisense Oligonucleotide AZD4785
}

National Cancer Institute

\section{Source}

National Cancer Institute. CEt KRAS Antisense Oligonucleotide AZD4785. NCI Thesaurus. Code C147521.

A proprietary formulation composed of a high affinity antisense oligonucleotide (ASO) that contains 2'-4' constrained ethyl residues (cEt) and targets KRAS (K-RAS) transcripts, with potential antineoplastic activity. Upon intravenous administration, cEt KRAS antisense oligonucleotide AZD4785 targets and binds, with high affinity, to a unique genetic sequence within KRAS messenger RNA (mRNA), thereby inhibiting translation of KRAS protein, including forms containing activating mutations. Inhibition of KRAS protein synthesis prevents KRAS-dependent signaling and inhibits the proliferation of KRAS-driven tumor cells. KRAS, a tumor-associated antigen (TAA), is mutated in a variety of tumor cell types. It plays a key role in tumor cell proliferation and survival and is associated with tumor initiation, metastasis and poor prognosis. 